\title{
La religione tra diritto e secolarizzazione
}

\author{
The religion between Law and Secularization
}

\author{
Francesco D'Agostino
}

Universitá di Roma «Tor Vergata» (Italia)

dagostino@lettere.uniromaz.it

RECIBIDO: 21/09/2017 / ACEPTADO: 23/10/2017

\begin{abstract}
Riassunto: II saggio presenta un'analisi critica dei fondamenti storici e teoretici della secolarizzazione, dimostrando l'inconsistenza dell'opzione secolarista (qualunque forma essa assuma: quella del «laicismo furioso», quella dell'agnosticismo morbido, o le varie versioni dei sostituti funzionali, tra cui in ambito giusfilosofico spicca il normativismo). La proposta è quella di tornare a semantizzare il diritto a partire da una visione del religioso e del sacro certamente aggiornati alla postmodernità, ma altrettanto certamente necessari in seno ad un discorso sul diritto e sui diritti che onestamente non eluda l'esigenza di un fondamento.
\end{abstract}

Parole chiave: secolarizzazione; fondazione del giuridico; religione.

\begin{abstract}
The essay presents a critical analysis of the historical and theoretical foundations of secularization, demonstrating the fragility of the secularist option (whatever form it takes: that of «furious laicism», that of soft agnosticism, or the various versions of functional substitutes, among which in jurisprudence there is normativism). The proposal is to go back to neutralizing the right from a religious and sacred vision, certainly up to postmodernity, but equally necessary in a speech about rights and rights that honestly does not circumvent the need for a foundation.
\end{abstract}

Keywords: secularism; foundation of legal system and rights; religion.

1. I tre termini che compongono il titolo di queste pagine sono banali a livello di linguaggio comune, ma irti di difficoltà a livello ermeneutico. Se infatti è già difficile attribuire un significato univoco al termine secolarizzazione e se è ancor più difficile (come già avvertiva Kant) definire il concetto di diritto, è difficilissimo (e probabilmente vano) introdurre un qualsivoglia riferimento al religioso che non appaia, oggi, scandaloso e che non induca dialoganti di buon senso a rimuoverlo accortamente dagli ordinari contesti dialogici, ad eccezione di quelli iper-specializzati o assolutamente protetti. Come parlare infatti del religioso in un orizzonte come quello del nostro tempo, che sembra non più in grado di coglierne la specificità e nel quale l'ostilità emotiva nei confronti della religione raggiunge spesso forme estreme e incontrollate ${ }^{1}$ ? Non minori

1 Se è perdonabile (sic!) il Nietzsche che afferma: «Contro il prete non si hanno ragioni: si ha il carcere!» (Prima proposizione della Legge contro il cristianesimo, in L'anticristo, tr. it. NIETZSCHE, F., Il Caso Wagner, Mondadori, Milano, 1975, p. 204) non lo è il poeta Edoardo Sanguineti, che alla 
sono però le difficoltà che oggi incontra un discorso sul religioso cui si voglia dare spazio sul piano, pur limitato, della mera filosofia. Se a livello scolastico e descrittivo la complessità implicita nel concetto di religione può in qualche modo essere controllata, è ben difficile ipotizzare la stessa cosa a livello rigorosamente teoretico. In una prospettiva, che è lecito qualificare come «classica» in senso ampio, quello che Hegel chiamava l'oggetto immenso del filosofare, cioè Dio stesso $^{2}$, si lascia toccare solo per rapide approssimazioni, per metafore, per allusioni (per i mistici solo per illuminazione), in quanto non è possibile ritenere di potersene impadronire. Ma, nell'orizzonte del pensiero oggi dominante al nome di Dio nulla corrisponde: «ma non nel senso che Dio, trascendendo ogni determinazione di essenza è non-ente o ni-ente, ma in quello dell'assolutamente nulla $\gg^{3}$. Solo pochi, tra coloro che amano porsi sulla scia di Nietzsche, riescono a capire che la sua istanza di fondo non è volgarmente ateistica: «la sentenza di Nietzsche: 'Dio è morto', non significa che Dio non esiste, perché questo implicherebbe ancora una sorta di tesi metafisica sulla struttura della realtà ${ }^{4}$, quando è proprio la questione se la realtà abbia una struttura che è in gioco in questo discorso. Queste difficoltà non possono essere superate, possono al più essere eluse. Le eluderò adottando due strategie diverse. Per la difficoltà che ho chiamato psicologica, non posso che invitare chi si senta in sintonia con i laici furiosi ${ }^{5}$, e pur tuttavia voglia continuare a leggermi, ad un eroico sforzo di pazienza. Invece allo svuotamento totale del Trascendente, a quella totale rimozione della metafisica cui corrisponde nell'epoca postmoderna il trionfo di una fisica assoluta, allo slogan heideggeriano Das Sein als Grund fabren lassen ${ }^{6}$, opporrò la categoria platonica del rischio: anche se è rischioso, anzi rischiosissimo, parlare di metafisica, dell'assoluto, di Dio, dell'essere - e questi concetti nell'ambito del mio discorso tendono a sovrapporsi, malgrado gli ammonimen-

domanda: Cosa detesta di più?, risponde con fermezza: La fede religiosa. Qualunque essa sia, è sempre espressione dell'infelicità umana (testimonianza raccolta da Paolo Di Stefano per il Corriere della Sera, 20.09.2013, Suppl. Io donna).

2 Philosophie der Religion, ed. Lasson, vol. I, Hamburg, Felix Meiner, 1966, p. 287.

3 Così CACCIARI, M., «Sull'ateismo ultimo», in AA.VV., Il cortile dei gentili, a cura di L. Mazas, Donzelli, Roma, 2011, p. 49.

4 VATtimo, G., «Quale futuro aspetta la religione dopo la metafisica?», in R. RORTY, G. VATTIMO, Il futuro della religione, a cura di S. Zabala, Garzanti, Milano, 2005, p. 68.

5 Traggo l'espressione dal titolo del bel libro di Bosetti, G., Il fallimento dei laici furiosi, Rizzoli, Milano, 2009.

6 Se ne vedano le ragioni e i limiti in VAтtimo, G., Addio alla verità, Meltemi, Roma, 2009, pp. 61 e ss. 
ti kantiani ${ }^{7}$, vale comunque la pena farlo, perché kalòs o kíndynos, perché si tratta di un rischio che è bello, anzi bellissimo, affrontare, come ci dice Platone (Fedo$n e, 114 \mathrm{~d})$. E tanto può bastare per dare inizio al mio discorso.

2. Partirò mettendo subito in chiaro che le considerazioni che andrò facendo si muovono lungo due linee argomentative, che esporrò secondo modalità inevitabilmente riduttive.

2.1. La prima linea argomentativa è di carattere teoretico e va in totale contro-tendenza rispetto al compatto e a-problematico fisicismo che caratterizza il post-moderno. Affermo che ogni sistema epistemologicamente dotato di senso (e quindi in particolare ogni sistema teorico-giuridico meritevole di attenzione) possiede -se ne sia o meno consapevoli- una matrice teologica, cioè assoluta: trova nel religioso le proprie radici. Parafrasando la nota affermazione di Wittgenstein: «Il senso della vita, cioè il senso del mondo, possiamo chiamarlo Dio» ${ }^{8}$, potremmo dire che «il senso dell'esperienza giuridica, cioè il senso del diritto, possiamo chiamarlo la giustizia di Dio». Si può negare un Dio, ma non si può negare Dio (con buona pace della National Secular Society e, in Italia, dell'Uaar, Unione degli atei e agnostici razionalisti). Si può essere atei nei confronti degli dèi del paganesimo o dell'induismo, o del Dio delle tradizioni monoteistiche; ma solo perché, implicitamente se non esplicitamente, si crede in una diversa divinità, cioè in un diverso fondamento dell'essere. Accettare questa affermazione in un orizzonte storico-etnologico è banale: la presenza del religioso in tutte le culture è un fatto; così come per gli storici e gli antropologi del diritto è un fatto che l'archetipo del giudice vada sempre rintracciato nella figura del «sacerdote». Il presupposto che sto esplicitando non ha però solo una valenza etnologico/fattuale; pretende di avere una valenza filosofica. Quando insisto nel rilevare che qualsivoglia comprensione del diritto (e di qualsiasi altro sistema epistemologico) richiede che se ne rilevi la matrice teologica (potremmo anche dire ontologica, dato che quest'ultimo aggettivo è più corretto, ma solo scolasticamente), intendo mettere in chiaro l'indisponibilità, da parte di chi voglia

\footnotetext{
7 Penso in particolare all'invito che Kant rivolge alla metafisica a deporre «l'orgoglioso nome di ontologia» (Critica della Ragion Pura, Analitica trascendentale, libro II, cap. III, tr. G. Colli, $3^{\circ}$ ed. riveduta, Adelphi, Milano, 1976, p. 320).

8 Notebooks, 1914-1916, nota dell'11.6.1916.
} 
studiarlo e fruirne, del fondamento del sistema: è in questa indisponibilità del fondamento che va ravvisata la dimensione del religioso'. Il fondamento (ogni fondamento) è di principio inaccessibile (se lo fosse, non sarebbe fondamento); dire però che è inaccessibile non significa dire che sia non percepibile o non nominabile $^{10}$; lo è, ma sempre in modo indiretto (nel cristianesimo, ad es., attraverso Cristo). Un altro modo di esprimere questo concetto è quello di affermare che ogni sistema, per essere tale, deve avanzare una specifica pretesa di verità (una pretesa a volte rozza e confusa, a volte limpida e sofisticata); una pretesa però e in ogni caso necessaria, come dimostra il fatto che coloro che rinunciano ad avanzarla inevitabilmente rinunciano ad ogni autentico impegno di carattere teoretico (e finiscono per accontentarsi spesso di surrogati, come quelli delle scienze umane: preziose quando in esse e attraverso di esse permane un intento teoretico, banali quando, private di tale intento, vengono ridotte a dinamiche meramente descrittive del darsi fenomenico dell'esperienza umana).

2.1.1. Esistono anche altre vie, alcune inaspettate, per illuminare questo presupposto e mostrarne la consistenza. Mi limito a citare la definizione luhmanniana della religione come della rappresentazione dell'appresentato ${ }^{11}$. Chi conosca un po' di fenomenologia, sa che Luhmann utilizza un fondamentale concetto elaborato da Husserl nella quinta delle sue Meditazioni cartesiane. Appresentazione per Husserl è la presenza alla coscienza dell'altro lato di un oggetto non direttamente percepibile, ma necessariamente co-presente, per la comprensione stessa dell'oggetto; essa è quindi il necessario complemento dell'oggetto (nel contemplare la Luna, devo implicitamente prefigurare nella mia mente che essa abbia un'altra faccia, inaccessibile alla mia osservazione, ma che devo necessariamente ipotizzare per avere della Luna una comprensione adeguata, come di un corpo celeste tridimensionale). Ciò che è presente lo è sempre assieme all'appresentato; in questo senso la religione accompagna tutta la nostra esperienza cognitiva, indicandocene il lato oscuro e nello stesso tempo garantendola come possibile e carica di senso.

9 Sto presentando in questo modo, in forma indebitamente compatta, l'epistemologia di Nicola Cusano, che viene oggi rivalutata come una delle più moderne che ci siano state trasmesse dal pensiero tardo-medievale. Si vedano le acute osservazioni di IsRAEL, G., «Il rapporto tra scienza e religione di fronte alle sfide della post-modernità», in Religione, scienza e la prova della ragione, a cura di G. Quagliariello, Cantagalli, Siena, 2008, pp. 65-66.

10 Cfr. Dalmasso, G., «Dio indicibile?», in Humanitas, 62, n 2 (marzo/aprile 2007), pp. 324-330.

11 Luhmann, N., Funzione della religione, tr. it., Morcelliana, Brescia, 1991. 
2.2. Per meglio qualificare ulteriormente questo mio punto di partenza, aggiungo che perfino le diverse e molteplici visioni scientifiche del mondo possiedono tutte un simile fondamento. Indipendentemente infatti da quanto ne siano consapevoli gli scienziati (e in genere, data la ristrettezza del loro orizzonte, ne sono ben poco consapevoli) tali visioni escludono in via preliminare (come appunto non può non fare ogni disciplina scientifica) ogni fondamento esoterico, magico, mistico, casuale, arbitrario, autoritativo, tradizionale di qualsivoglia forma di sapere per la quale si rivendichino i caratteri della consistenza e della comunicabilità. Infatti, quanto più un sapere si pone come rigoroso, tanto più pretende di essere riconducibile a leggi empiricamente accertabili $\mathrm{o}$ almeno razionalmente ipotizzabili (come nel caso del paradigma evoluzionistico darwiniano) e, in quanto tali, assolute (non nel senso che non siano riformulabili, ma nel senso che non possono esserlo arbitrariamente). Il celebre motto che riassume il pensiero del maggior filosofo positivista italiano, Roberto Ardigò: il fatto è divino, va compreso non come un'ingenua metafora, ma come espressivo di un bisogno di ontologia (peraltro drammaticamente destinato a restare senza adeguata risposta in una prospettiva piattamente positivistica).

2.2.1. Nei sistemi sociali e in particolare in quelli giuridici il fondamento teologico che li sorregge può essere variamente denominato (e a volte sono richiesti faticosi lavori filologici per farne emergere la valenza). A parte i sistemi giuridici che utilizzano esplicitamente il nome di Dio per corroborare il loro fondamento ${ }^{12}$, potremmo menzionare la categoria del bene comune come il fondamento dei sistemi societari, quella della sovranità come il fondamento dei sistemi politici, quella della bellezza come il fondamento delle pratiche estetiche, quella della salvezza come il fondamento delle religioni istituzionalizzate, quella della verità come il fondamento (problematico?) dei sistemi filosofici, ecc. I sistemi si intrecciano e si fecondano reciprocamente, poiché il fondamento di ognuno di essi non è autoreferenziale, ma costituisce un versante di ciascun altro: ipotizzare il contrario, cioè ammettere sì che esista una molteplicità di fondamenti, ma considerarli irrelati, al fine di indebolirne la valenza, per sottrarre loro ogni pretesa di assolutezza (e al limite per svuotarli di senso), non significa liberarsi dalla metafisica o dalla religione, ma piuttosto cadere nel politeismo ontologico e assiologico ${ }^{13}$.

\footnotetext{
12 Studiati acutamente da CoTTA, S., «Il nome di Dio nel linguaggio giuridico», in ID., Itinerari esistenziali del diritto, Morano, Napoli, 1972, pp. 147 e ss.

13 WEIL, S., La prima radice, tr. it., Milano, 1954, p. 268.
} 
2.2.2. Poiché dobbiamo dedicare la nostra attenzione al nesso diritto/ religione, possiamo subito rilevare come nei confronti del diritto la religione assolva ad una funzione molto precisa, quella di dare un fondamento non arbitrario alla giustizia e che reciprocamente il diritto assolve nei confronti della religione ad una funzione altrettanto rilevante, poiché la garantisce dal rischio, sempre immanente nelle religioni istituzionalizzate, di degradarsi, avallando pratiche ciecamente violente ${ }^{14}$. Utilizzando un'efficace formula sociologica, potremmo dire che in un sistema giuridico (ma più in generale in tutti i sistemi sociali) la religione assolve ad una funzione di Kontingenzbewältigungspraxis: essa assolve cioè ad una funzione di superamento della contingenza. Essa però può adempiere a questa funzione solo quando la legge, il sacro filo della ragio$n e^{15}$, le impedisce di cedere all'ordine delle emozioni ${ }^{16}$.

2.2.3. E' indispensabile superare la contingenza? Assolutamente sì, sia a livello cognitivo che a livello valoriale. A livello cognitivo, non è possibile elaborare alcun sapere, se non si ha la possibilità di porre un nesso, cronologico e logico, tra gli eventi, trascendendone quindi la contingenza e rilevandone le reciproche concatenazioni. A livello valoriale, superare la contingenza significa qualificare l'impegno degli uomini nel mondo come dotato di senso e fornire adeguate ragioni ed esplicazioni per le nostre decisioni: per quelle scelte, cioè, di intervenire nel mondo tagliandolo e ritagliandolo (perché non altro significa propriamente il verbo decidere se non tagliare), umanizzandolo e rendendolo quindi diverso da come si presenta ai nostri occhi nella sua inerzia costitutiva. Peraltro, agli uomini non si offre altra scelta: il mondo è come la testa di Medusa, il cui sguardo pietrifica chi non sia in grado di tagliarla, chi cioè non sia in grado di operare su di essa decidendo. E' un'illusione ritenere di poter costruire un'adeguata teoria del diritto ponendo a suo fondamento la decisione. In tal modo infatti non si elude la questione teoretica fondamentale: essendo esposta al rischio mortale della contingenza, e quindi dell'insignifican$z a$, e quindi della paralisi, ogni decisione ha bisogno di essere salvata, di trovare cioè un fondamento su cui radicarsi. Il decisionismo giuridico e la sua forma più estrema, il nichilismo giuridico, sono le più fragili delle letture del diritto.

\footnotetext{
14 Del che era ben consapevole Gesù Cristo: «Viene l'ora in cui chiunque vi ucciderà crederà di rendere culto a Dio» $(\mathrm{Gv}, 16.2)$.

15 Platone, Leggi, 645a.

16 Platone, Repubblica, X.606-607.
} 
2.3. La seconda linea argomentativa del mio discorso è di carattere sociologico-culturale. Ritengo che si possa sostenere che tutta la storia della filosofia (in particolare nell'epoca moderna) appare dominata da una straordinaria ed enigmatica tensione, o, se così si vuol dire, da un irriducibile desiderio, quello di negare l'assoluto e di dichiararlo illusorio, fraudolento, irrilevante, pericoloso, infantile, teoreticamente inconsistente o comunque meritevole di radicale trasfigurazione (Umwertung aller Werte). Dare un'adeguata spiegazione di questa tensione va al di là dei limiti di questo discorso e delle mie stesse capacità: ma non voglio perdere l'occasione di osservare come questo - soprattutto se letto come tema teologico, come io credo sia indispensabile fare -sia uno dei temi assiali della storia universale. George Steiner ci dà questa indicazione a suo modo ${ }^{17}$, osservando come gli ebrei siano perseguitati non per aver ucciso Dio, ma per averlo inventato.

2.4. L'espressione più compiuta di questa tensione si ha con l'illuminismo, in pressoché tutte le sue varianti. Possiamo serenamente sostenere che nella sua insistente critica del religioso, l'illuminismo abbia raccolto una significativa messe di fallimenti ${ }^{18}$. E' inutile soffermarsi sulle forme più primitive dell'illuminismo (ancor oggi riproposte), quelle che vedono nella religione un subdolo inganno attivato dai preti o dai potenti di turno per meglio sfruttare la credulità delle masse e ottenerne facile ossequio, quelle che riducono il sentimento religioso alla manifestazione di una nevrosi, senza percepire come il bisogno di credere sia altrettanto autentico e antropologicamente costitutivo del desiderio di sapere $^{19} \mathrm{o}$ quelle, solo relativamente più sofisticate, che vedono nella religione una dinamica di etica popolare, destinata a evaporare quando posta a confronto con forme di etica illuminata o di etica filosofic $a^{20}$ : non è una confutazione della religio-

17 SteINER, G., I libri che non ho scritto, tr. it., Garzanti, Milano, 2008, p. 130.

18 LÜBBE, H., «Religion nach der Aufklärung», in ID., Philosophie nach der Aufklärung, Econ, Düsseldorf-Wien, 1980, pp. 59-85, tr. it., La religione dopo l'illuminismo, Morcelliana, Brescia, 2010.

19 KRISTEVA, J., Cet incroyable besoin de croire, tr. it., Bisogno di credere. Un punto di vista laico, Donzelli, Roma, 2006, pp. 5 e ss. e, più recentemente, «La grammatica del credere», in Vita e Pensiero, $\mathrm{n}^{\circ} 3$ (2011), pp. 74 e ss. Cfr. anche, sempre della Kristeva, «Osare l'umanesimo», in Il cortile dei gentili, cit., pp. 18-19.

20 Si rilegga la lettera del dicembre 1949, nella quale Benedetto Croce, rivolgendosi al politico cattolico Alcide De Gasperi, lo conforta paternalisticamente, invocando su di lui l'aiuto di Dio «Perché anch'io credo, a modo mio, a Dio, a quel che a tutti è Giove», scrive il filosofo adottando un'espressione filosoficamente lieve, ma non imprecisa, per evitare evidentemente concetti da lui ritenuti non alla portata della semplice mente del suo interlocutore. La lettera è riportata quasi per intero su «Avvenire» del 16.6.2012, nell'articolo di DE GASPERI, M. R., E Don Benedetto disse ad Alcide: «Che Dio ti aiuti...». 
ne una morale che si trasforma (come sembra avvenga in Kant) nella religione di coloro che dichiarano di non aver religione, ma una sua paradossale conferma. A tutte queste posizioni basterebbe opporre i lucidi sarcasmi di Lacan, quando, dopo aver rilevato quanto poco si sia consapevoli della forza del religioso, ne diagnostica (o ne profetizza?) l'ultimativo trionfo ${ }^{21}$. Merita piuttosto una sia pur breve attenzione la pretesa secondo la quale il processo di secolarizzazione comporterebbe il progressivo e irreversibile declino dell'autorità religiosa sulle cose del mondo e una necessaria e progressiva implosione del religioso.

3. Si può affermare che tutta la sociologia della religione del Novecento (ma potremmo rifarci anche alla seconda metà dell'Ottocento e citare un nome come quello di Durkheim) si fonda su questo dogma, ampiamente smentito dalle ricerche degli ultimi decenni ${ }^{22}$. Il processo di secolarizzazione (peraltro non omogeneo nelle diverse culture, né riconducibile a stilemi univoci) ha prodotto (ma non sempre) dinamiche di desacralizzazione, ma non ha attivato, di per sé, un affievolimento del religioso in quanto tale. Alla crisi della religiosità istituzionale (peraltro anch'essa non riducibile a dinamiche omogenee) non corrisponde statisticamente una crisi della religiosità personale (in termini lievemente più tecnici, alla crisi del Belonging non corrisponde una crisi del Believing): l'allentamento del legame tra credenza e appartenenza non comporta l'affievolimento del religioso, quanto piuttosto il sostituirsi alla figura del credente praticante di quella del credente pellegrino, che presume di poter produrre da sé i significati della propria esistenza nella diversità delle situazioni che volta per volta va sperimentando ${ }^{23}$, riducendo di fatto la scelta del religioso a una mera preferenz $a^{24}$. In altre parole la deregulation del religioso ha sociologi-

21 LaCan, J., Dei Nomi-del-Padre seguito da Il trionfo della religione, testi riuniti da J.A. Miller, tr. it., Einaudi, Torino, 2006, p. 98.

22 Una prima, efficace rassegna di queste smentite è nel libro di STARK, R.; INTROVIGNE, M., Dio è tornato. Indagine sulla rivincita delle religioni in Occidente, Piemme, Casale Monferrato, 2003. Recentissimo l'intervento di SADUN BORDONI, G., Religion in a post-secular World, in «Longitude», (January 2012), pp.72-77.

23 Davie, G., Religion in Britain since 1945, Blackwell, Oxford, 1994, cit. da Hervieu-LÉGER, D., «Cristianesimo e post-modernità. Il cattolicesimo», in G. Filoramo (a cura di ), Le religioni e il mondo moderno, vol. I, Einaudi, Torino, 2008, p. 572.

24 E' questa la nota e inconfutata tesi di Berger, P., The Far Glory. The Quest for Faith in an Age of Credulity, Free Press, New York, 1992, tr. it., Una gloria remota, Il Mulino, Bologna, 1994. Utilissime le considerazioni che a partire da questo libro sono svolte da Perniola, M., Del sentire cattolico. La forma culturale di una religione universale, Il Mulino, Bologna, 2001. 
camente ben poco a che vedere col suo affievolimento. In questo consisterebbe, secondo Ulrich Beck, il paradosso della secolarizzazione: all'indebolimento terreno delle religioni sarebbe da attribuire la ragione della loro rinnovata vitalità. Scrive Beck: «Proprio nella società secolare le religioni 'derubate' del rilevante ruolo di forgiare le coscienze -separazione tra religione e scienza- e di legittimare il potere -separazione tra religione e Stato- possono ottenere una nuova funzione e autorità in quanto scuola di morale» ${ }^{25}$.

4. Alla profezia sociologica del progressivo deperimento e dell'estinzione definitiva delle religioni nel contesto della modernità -rivelatasi infondata- si affianca l'altrettanto infondata pretesa, sociologico-politica, di affrettarne l'agonia, escludendole radicalmente dalla sfera pubblica. Tale esclusione è giustificata essenzialmente dal convincimento che il religioso sia incompatibile con la modernità in generale e con la modernità globalizzata in particolare: attivando dinamiche di radicamento identitario, il religioso produrrebbe inevitabilmente forme irriducibili di conflitto. Riprendendo un'opinione diffusa le religioni sarebbero pericolose. Nessuno più dei giuristi può apprezzare la profondità di questa diagnosi (indipendentemente dalla sua condivisibilità): la costante, sistematica, pericolosa epurazione dai sistemi e dal linguaggio giuridico di ogni riferimento al religioso (fino addirittura alle sue epifanie marginali, come quelle che si manifestano ad es. nel giuramento, sempre più sottoposto ad un'erosione sistemica) potrebbe esserne una significativa conferma.

5. Nella prospettiva che sto difendendo, bisognerebbe invece riconoscere che al fallimento fattuale delle teorie sociologico-religiose della secolarizzazione (fallimento, come già detto, ormai ampiamente verificabile) corrisponde un altrettanto palese fallimento delle pretese valorative teorico-dottrinali del processo di secolarizzazione. L'espressione secolarizzazione della secolarizzazione, che oggi va sempre più diffondendosi, è indicativa di un fatto che non può più essere negato: il processo di secolarizzazione appare in crisi irreversibile, non tanto perché si assista ad un revival del religioso (anche se ci sono studiosi che autorevolmente lo sostengono), ma perché si tratta di un processo che non è stato in grado di mantenere la sua promessa essenziale, quella di sostituire

25 BECK, U., Il Dio personale, tr. it., Laterza, Roma-Bari, 2009, p. 155. 
alla salvezza extra-mondana annunciata dalle religioni una ben più corposa salvezza all'interno di questo mondo, l'unico nel quale -per gli antimetafisici secolarismi-gli uomini si trovano a vivere. In altre parole, siamo tutti testimoni dell'incapacità di qualsivoglia sistema sociale secolarizzato a generare forme vitali di coesistenza, capaci di sostituire funzionalmente quelle strutturate dalle religioni. Sottraendo a Dio la fonte legittima dell'autorità politica, per attribuirla al popolo, abolendo la trascendenza per dare al sociale un fondamento assolutamente immanente, l'illuminismo non ha vinto la sua battaglia, ma ha innestato un'interminabile serie di crisi, che negli ultimi due secoli hanno assunto aspetti polimorfi, ma nell'essenziale non divergenti (nell'Ottocento il nazionalismo e il colonialismo, nel Novecento il fascismo, il nazismo e il marxismo-leninismo, nel Duemila il liberalismo dei sistemi finanziari). In altre parole la modernità, illudendosi di essere in grado di razionalizzare le radici dei sistemi sociali, ha operato in senso assolutamente contrario; non è riuscita ad altro se non a porre al posto di un principio ontologico ardito, ma razionale, come appunto quello di logos, come appunto quello di Dio o di assoluto, concetti mitologici, cioè pseudo-religiosi e sostitutivi, come quelli di nazione, popolo, classe, capitale, ordinamento giuridico (utilissimi in contesti epistemologici ristretti - politica, storia, economia, teoria del diritto, ecc.- ma privi di una portata veritativa) o al massimo concetti etici che, depauperati di un fondamento ontologico, si rivelano fragilissimi (ancorché nobili), come quelli di umanità, solidarietà, fraternità ${ }^{26}$.

6. Per i giuristi la migliore esemplificazione di quanto stiamo dicendo ce la può fornire una breve riflessione sul sistema kelseniano. Anche se da lui presentato come un'esigenza logica, quel fondamento del sistema giuridico che è per Kelsen la norma fondamentale è un autentico sostituto della volontà normativa della natura o di Dio: oggi ci appare come un concetto palesemente mitologico, che dimostra, sia pur indirettamente, l'irrinunciabilità per ogni sistema giuridico di un presupposto ontologico (un Ardigò lettore di Kelsen avrebbe di certo sostenuto che la norma fondamentale è divina). In chiave non ontologica, ma sociologi-

26 «In termini di linguaggio simbolico, si può dire che la bilancia e la spada hanno progressivamente cessato di essere guidate dalla mano di Dio e si sono affidate a forme di religione secolarizzata - il popolo, la nazione, la classe e così via - senza che si sia riusciti a risolvere il problema di come dotare quei simboli dell'antica certezza religiosa del giusto» (così ProsPERI, A., Giustizia bendata, Einaudi, Torino, 2008, p. 227). 
ca, il risultato ultimo del kelsenismo è stato quello, come aveva perfettamente compreso Giuseppe Capograssi ${ }^{27}$, di aver reso terribilmente difficile almeno per i normativisti l'elaborazione di un'adeguata teoria dell'obbligo giuridico (perché una norma fondamentale dovrebbe obbligare gli esseri umani, quando questi non si sentono, in genere, obbligati nemmeno dalla volontà divina?). L'esito di questa e di simili sostituzioni è sotto gli occhi di tutti: ad es. se il rendere giustizia in nome di Dio poteva e può continuare ad apparire tragicamente presuntuoso, il rendere giustizia in nome del popolo appare oggi tragicamente elusivo, per la nostra incapacità di qualificare con rigore razionale l'identità popolare. Per il giurista la conferma di quanto stiamo dicendo si manifesta nel crollo di una grande e generosa illusione, quella della possibilità di istituire un sistema non solo giuridico, ma soprattutto giurisdizionale, mondiale, capace di garantire la libertà e i diritti umani di tutti e per tutti. Alcuni per la verità si consolano sostituendo alla pretesa di un obbligo giuridico planetario la ben più limitata pretesa di individuare un fondamento per gli obblighi politici «regionali», postulando il diritto di ogni popolo di essere governato a partire da un sistema costituzionale comunque rispettoso dei diritti umani. Ora, si può ben individuare nella Costituzione il simbolo di una legge superiore e delle forze unificanti che operano in una comunità per tenerla unita; ma se ai simboli si dà un fondamento irrazionale, tutto ciò che su di essi si vuole fondare acquisterà inevitabilmente una dimensione pericolosissima di irrazionalità. Postulare la libertà come valore sociale supremo non ha di certo nulla di irrazionale; ma la libertà chiede di essere fondata, non di essere semplicemente espressa simbolicamente, come nell'immensa tela, La Libertà guida il popolo, dipinta nel 1830 da Delacroix. Non ci è concesso, in buona sostanza, dimenticarci del monito di Tocqueville, per quanto duro possa apparire a un laicismo narcisista: «L'uomo, se non ha fede, bisogna che serva; e, se è libero, che creda $»^{28}$. Un monito, questo, che si pone nella stessa linea di una nota -e spesso fraintesa- osservazione hegeliana (anche se probabilmente Tocqueville non ne era consapevole), quella secondo la quale «a Platone non fu dato di poter progredire fino a dire che finché la vera religione non entrerà nel mondo e non diventerà dominante negli Stati, il vero principio degli Stati non sarà pervenuto alla sua realtà $\gg^{29}$.

\footnotetext{
27 Si rilegga il suo classico saggio Impressioni su Kelsen tradotto, Giuffrè, Milano, 1952, poi in Opere, Giuffrè, Milano, 1959, vol. V, pp. 313-356.

28 Tocqueville, De, A., La Democrazia in America, tr. it., Torino 1968, p. 510.

29 HeGEL, G.W.F., Enciclopedia delle scienze filosofiche in compendio, $\$ 552$.
} 
7. Il punto è che non esistono equivalenti funzionali della religione, così come non esistono equivalenti funzionali del principio di giustizia. Per il giurista questo dovrebbe ormai essere evidente, perché gli esempi in materia sono innumerevoli. La secolarizzazione ha operato con forza per il passaggio dal giuramento come contratto garantito dalla divinità al giuramento come sacralizzazione della politica e del potere, in nome di una nuova religione civile ${ }^{30}$ : tanti sforzi non hanno di certo contribuito a mantenere al giuramento l'aura di sacralità che lo distingue dalla mera promessa e ne hanno, anzi, affievolito pressoché definitivamente la valenza. Insomma, l'ingenuità di chi ritiene che «il nichilismo ci salva e ci protegge» e costituisce una «medicina nei confronti di antichi e nuovi morbi: unità, totalità, globalità, universalità», smascherando ulteriori «falsi idoli» ${ }^{31}$ ce ne dà la prova, perché è evidente che chiunque insista ancora nel parlare della morte di Dio dovrebbe pur riconoscere l'avvenuta e irreversibile morte di Nietzsche. E che di morte si tratti, e per di più di una morte priva di resurrezione, dovrebbe ormai essere palese agli occhi di tutti.

8. L'annuncio della morte di Dio non ha prodotto una Umwertung aller Wer$t e$, ma ben più banalmente il dilagare sul piano dei principi di un relativismo scettico, dell'una cosa vale l'altra, quel relativismo bonariamente sbeffeggiato da Chesterton (ma probabilmente la citazione è apocrifa): chi non crede in Dio, pensa di non credere in nulla, mentre invece, anche se non se ne accorge, crede letteralmente in tutto, in tutto e al contrario di tutto. Bisogna attivare una terapia contro questo eccesso scettico, contro «l'atteggiamento, celebrato come una virtù, degli indifferenti che oggi imperversano, un atteggiamento spesso mascherato da eccessive e troppo zelanti professioni di fede che non costano niente e consentono perciò facili, spregiudicati cambiamenti di fronte, preludio di immorali alleanze per fame e sete non di giustizia, ma di potere e successo personale ${ }^{32}$ ? Indubbiamente sì, anche per evitare che col termine

30 Cfr. Prodi, P., Il sacramento del potere, Il Mulino, Bologna, 1992, p. 423.

31 Così IrTi, N., Nichilismo giuridico, Laterza, Roma-Bari, 2004, p. 148. Chi ritenga troppo frettolosa la riflessione condotta nel testo, può utilizzare con profitto le analisi critiche di PosseNTI, V., Nichilismo giuridico. L'ultima parola?, Rubbettino editore, Soveria Mannelli, 2012, pp. 127 ss. o quelle di Galantini, L.; Palmaro, M., Relativismo giuridico. La crisi del diritto positivo nello Stato moderno, Vita e Pensiero, Milano, 2011.,

32 Così Zagrebelsky, G., L'idea di giustizia e l'esperienza dell'ingiustizia, in MARTINI, C. M.; ZAGREBELSKY, G., La domanda di giustizia, Einaudi, Torino, 2003, p. 49. 
relativismo si finisca per indicare l'esito necessario e conclusivo della tradizione liberale ${ }^{33}$. E' con questo intento che molti si pongono alla ricerca delle modalità ottimali di fondazione di un'etica pubblica, individuandole nell'indifferenza (se non nell'ostilità) nei confronti delle dinamiche religiose e nell'appoggio esplicito al paradigma dei diritti umani, come traduzione post-metafisica e anti-ideologica del principio di giustizia. Il limite di tale tentativo consiste in questo, che per costruire tale etica bisogna necessariamente darle un carattere plurale (per l'impossibilità di una lettura univoca delle tavole dei diritti), ma questa pluralità, anziché garantire la salvezza dal relativismo nichilista, torna inevitabilmente a riproporlo, poiché produce inevitabilmente dinamiche conflittuali perpetue e insolubili ${ }^{34}$. La prova di quanto detto emerge con chiarezza in tutti quei casi in cui il pluralismo etico viene davvero messo alla prova sul piano pubblico: si pensi agli interminabili dibattiti -che si trasformano a volte in scontri molto duri- sulla libertà di espressione, di stampa e di satira, sui diritti delle donne, sulle mutilazioni sessuali non solo femminili, ma anche maschili, sul riconoscimento legale dei tabù alimentari, fino alle stesse strutture individualistiche e non comunitarie della democrazia formale occidentale.

9. Ecco perché non deve stupire che alla crisi del religioso indotta (e mal governata o non governata affatto) dalle dinamiche della secolarizzazione abbiano fatto seguito non forme nuove e inedite di liberazione psicologico-sociale del genere umano (vanamente preconizzate dagli illuministi), ma inattese, inquietanti e spesso tragiche forme di asservimento mitico/ideologico. Alludo alla proliferazione delle credenze più ingenue, al diffondersi delle sette, al riproporsi di grotteschi pregiudizi e di antiche superstizioni, alla ricomparsa di un paganesimo ecologicamente e ciecamente orientato, al consolidarsi di forme di cieca idolatria nei confronti della scienza e soprattutto al radicarsi prima, e all'esplodere poi del fondamentalismo (un'esperienza para-religiosa, pressoché sconosciuta nella storia). Il tutto nel contesto di un mondo globalizzato, reso strutturalmente insicuro da vorticose crisi identitarie e dalle ingenue risposte elaborate per fronteggiarle (solo chi abbia visitato e osservato da vicino le immense metropoli di quello che un tempo era chiamato il Terzo Mondo - da

33 Cfr. VAtтimo, G., Addio alla verità, cit., p. 60.

34 Lo dimostra, con abbondanza di analisi, DONATI, P., La matrice teologica della società, Rubbettino editore, Soveria Mannelli, 2010, in part. pp. 61 e ss. 
Città del Messico a Manila - può davvero comprendere a cosa ci si riferisca quando si parla, a livello urbano, di insicurezza). A questa insicurezza bisogna dare una risposta antropologica, capace di manifestarsi in forma adeguata sia sul piano politico che su quello giuridico: a questa consapevolezza stanno, sia pur lentamente, giungendo quei politici e quei giuristi che, avendo preso atto con onestà intellettuale del fatto che la completa laicizzazione del politico e del giuridico esige nuove integrazioni di senso, riconoscono non solo l'opportunità, ma la necessità di dare nuova visibilità pubblica al religioso ${ }^{35}$.

10. Il nostro problema è a questo punto simile (anche se non del tutto riducibile) a quello che si pone da tempo Jürgen Habermas: come governare il pluralismo postmoderno e -il che è esattamente lo stesso problema- come riammettere il religioso nel foro pubblico (politico e giuridico)? Una volta purificatolo dalle scorie che ne deformano l'identità e una volta rimossa l'ideologia che lo percepisce come un relitto arcaico di cui sbarazzarsi definitivamente, come tornare a reinserire il religioso nel sociale come forza postmoderna? L'irriducibile molteplicità del religioso non costituisce un ostacolo insuperabile alla realizzazione di questo obiettivo?

11. Il problema del pluralismo religioso viene spesso interpretato come un problema immensamente rilevante, sì, ma comunque storicamente condizionato e spesso (anche se inconsapevolmente) riducibile a una questione di ecologia culturale (come ad es. quella della difesa delle lingue minoritarie, minacciate di scomparsa). Non è così: quello del pluralismo è piuttosto un problema antropologico. Il pluralismo infatti si presenta come esaltazione delle differenze culturali sia a livello macro (i valori propri delle singole etnie) che a livello micro (gli stili di vita personali), ma nel contempo nell'orizzonte pluralistico postmoderno le differenze vengono banalizzate, attraverso il postulato dell'assoluta eguale sovranità dei diritti umani universali: si crea così quella che è stata chiamata un' indifferenza delle differenze, che produce crisi identitarie profonde e insanabili. Infatti, nel sistema multiculturale globalizzato, gli esseri umani sono nel tempo stesso tutti differenti e tutti eguali: la cosa crea un paradosso irresolubile. E' questa la ragione per la quale l'unica via di uscita ritenuta

35 Belardinelli, S., Sillabario per la tarda modernità, Cantagalli, Siena, 2012, pp. 131 e ss. 
praticabile da molti appare quella autoritaria (come ad es. nel caso esemplare e irrisolto, perché irrisolubile, della repressione penale introdotta di recente in alcuni ordinamenti della circoncisione dei neonati e delle mutilazioni sessuali femminili, ancorché su donne competenti e consenzienti). Si osservi ancora che il pluralismo religioso, come fatto, non solo conferma la matrice teologica del politico e del giuridico, ma rappresenta l'esito dell'impressionante frantumazione e dilatazione operata dalla cultura moderna di poche essenziali matrici in una molteplicità di epifenomeni. Di qui il senso di disgregazione che sembra qualificare la post-modernità, una disgregazione che si fa storia, a seconda dei contesti, non solo nell'ostilità etnica e nel confronto conflittuale di fedi e di riti, ma anche nell'implosione demografica, nell'invasività tecnologica, negli smarrimenti bioetici, tutte dinamiche espressive di una crisi antropologica profonda.

12. Ritorniamo alla domanda fondamentale: come tornare a reinserire il religioso nel sociale come forza postmoderna? Come attivare nelle società post-secolari dinamiche di apprendimento complementare tra il giuridico e il religioso ${ }^{36}$ ? La via da percorrere sembra obbligata ed è riassumibile in questi termini. Sia nella sua dimensione funzionale minima, quella che, come si è detto, i sociologi qualificano quale Kontingenzbewältigungspraxis, sia nella sua dimensione funzionale massima, che nel cristianesimo si presenta come assolutizzazione del principio di fraternità («Ama il prossimo tuo come te stesso»), il religioso -al di là delle sue molteplici configurazioni confessionali- opera come superamento della tentazione individualistica in cui il post-moderno trova la sua realizzazione più compiuta e dona fondamento adeguato a quella logica della $c 0-$ municazione che va oltre l'indifferenza delle differenze e che anzi fa del rispetto delle differenze il presupposto stesso del comunicare. La globalizzazione ci dimostra che nel nostro tempo, diversamente che in passato, l'individuo può senza contraddizione assumere molte identità: l'importante è che «le differenze tra le identità siano trattate relazionalmente, il che significa vedendo l'universale nelle/con/attraverso le differenze» ${ }^{37}$. Quanto più i sistemi giuridico-politici

\footnotetext{
36 Forzo, ma molto lievemente, le problematiche poste sul tappeto da HABERMAS, J.: cfr., in particolare, I fondamenti morali prepolitici dello Stato liberale, tr. it. in HABERMAS, J. e RATZINGER, J., Etica, religione e Stato liberale, Morcelliana, Brescia, 2004, p. 37.

37 Donati, P., La matrice teologica della società, cit., p. 220.
} 
adempiranno al loro dovere di aiutare le religioni a liberarsi da superflui vincoli identitari di carattere etnico, tanto più otterranno come ricompensa (se così si può dire) la possibilità di dare quel fondamento adeguato (cioè non arbitrario, non convenzionale, non formalmente e superficialmente simbolico) all'ordine degli obblighi giuridico-politici di cui si avverte assolutamente il bisogno. In un mondo globalizzato, il punto di incontro tra le diverse culture non può più essere affidato agli Stati e ai loro ordinamenti giuridici particolari, né all'universale astratto dei diritti umani presentati nella loro formulazione illuministica, né alla comunità illimitata di discorso su cui tanto insiste Habermas, e nemmeno, più in generale, a quelle innumerevoli proposte generosamente ma anche ingenuamente dialogiche che si manifestano fin dall'inizio come pura retorica ${ }^{38}$ : bisogna piuttosto declinare l'universale nel rispetto delle innumerevoli semantiche particolari, purché queste siano tutte percepite come espressioni dell'bumanum e del bene che ne costituisce l'essenza. Il principio costitutivo di tutte le religioni, l'essere l'uomo capax Dei, fornisce l'unica chiave a nostra disposizione per la costruzione di un fattivo universalismo. Quella che è stata presentata dalla migliore teologia contemporanea come sintesi tra fides e ratio, una sintesi che qualifica, sia pure in forme diverse, tutti i grandi monoteismi (e quello cristiano in particolare) garantisce che all'interno delle società civili contemporanee si possano attivare forme di comunicazione non escludenti, capaci quindi di garantire il principio stesso del diritto. Si può ottenere questo risultato senza pretendere che queste società debbano rinunciare, ancorché in minima parte, alla loro laicità (cioè al pari rispetto per tutte le confessioni religiose e per tutti gli orizzonti valoriali); è sufficiente che non abbiano paura di essere religiosamente qualificate, perché solo in tal modo si può evitare l'esito catastrofale di una modernità che ceda alla tentazione di strutturare sistematicamente l'irreligiosità, negando il fondamento assoluto dei diritti, della giustizia, del bene comune. Questa negazione, cioè, in altre parole, il consolidarsi di una società in cui sia spento il senso di giustizia, equivarrebbe alla legittimazione di una società disumana, in cui non vale la pena vivere. Con queste espressioni di Gustavo Zagrebelsky, che emergono da un suo ormai lontano, appassionato dibattito col Card. Martini, appare opportuno concludere queste riflessioni ${ }^{39}$.

38 VAттімо, G., Della realtà. Fini della filosofia, Garzanti, Milano, 2012, p. 220.

39 Martini, C. M.; Zagrebelsky, G., La domanda di giustizia, cit., pp. 67-68. 